\title{
Using 50-mm electrostatic membrane deformable mirror in astronomical adaptive optics
}

\author{
Andrei Tokovinin ${ }^{a}$, Sandrine Thomas $^{a}$, Gleb Vdovin ${ }^{b}$ \\ ${ }^{a}$ Cerro Tololo Inter-American Observatory, Casilla 603, La Serena, Chile \\ ${ }^{b}$ Electronic Instrumentation Lab, TU Delft, \\ Mekelweg 4, 2628 CD Delft, The Netherlands
}

\begin{abstract}
Membrane micro-machined deformable mirrors (MMDM) feature low cost, low power consumption, small size and absence of hysteresis. Interested in using such a device for the adaptive optics system at the SOAR 4.1-m telescope, we evaluated the performance of a 79-channel 50-mm (pupil size 35mm) MMDM from OKO Technologies. The measured influence functions match the solutions of the Poisson equation with a fixed boundary. The maximum achievable modulation of the local radius of curvature of the mirror is $\pm 25 \mathrm{~m}$. Simulations of the AO system with this MMDM show that the curvature saturation becomes apparent even at a median seeing of 0. .'67. A new control algorithm that re-distributes the signals of saturated electrodes to adjacent ones significantly improves compensation under conditions of partial saturation. Limited curvature range and pupil diameter restrict the suitability of MMDM technology to moderate order AO systems at telescopes with diameter of up to $3 \mathrm{~m}$.
\end{abstract}

Keywords: Adaptive optics, Deformable Mirrors, Atmospheric Correction

\section{WHY ELECTROSTATIC MEMBRANE DM?}

Maturing of the Adaptive Optics (AO) technology leads to its wider use in astronomy and other fields. The availability of relatively inexpensive and reliable key components, such as Deformable Mirrors (DMs), is crucial for the AO accessibility. In this respect, membrane electrostatic DMs from Okotech ${ }^{1}$ beat the record of price per actuator. In these devices, a thin membrane is stretched over a board with printed electrodes. A voltage difference between the membrane and those electrodes creates an attraction force roughly proportional to the square of the voltage and generates local curvature of the membrane over the electrode. The difference between the two types of curvature DMs - electrostatic and bimorph - is in the boundary conditions: a bimorph DM usually has a free edge, whereas a membrane DM has a fixed edge. Membrane DMs do not have hysteresis and are sufficiently fast for adaptive correction of turbulence. They need low driving current and hence consume low power.

Electrostatic DM from OKO Technologies with 37 electrodes (hereafter DM-37) is used in the AO system at McMath-Pierce solar telescope ${ }^{2}$ and at the $1 \mathrm{~m}$ telescope. ${ }^{3}$ We considered this technology attractive for an AO instrument at the 4.1-m SOAR telescope. ${ }^{4}$ A larger number of actuators is needed, so we select a DM with 79 electrodes and 50-mm membrane (DM-79). These devices were originally developed for space applications. ${ }^{5}$ Only the central part of the membrane (35 $\mathrm{mm}$ in diameter) can be controlled, the outer part being a transition zone to the fixed edge. Such a small size with a large number of electrodes are unique features of DM-79 that enable a compact design of the AO instrument.

Like any curvature DM, electrostatic membrane DMs are limited in maximum curvature, rather than in surface deformation (stroke). Specification of dynamic range for curvature DMs is thus non-trivial. Previous experience with such DMs have indicated that saturation can be a problem. ${ }^{6}$ Our initial estimates for DM-79 working at 4.1-m telescope gave an alarming result that the saturation limit can be approached even under median seeing conditions. However, the limits of membrane technology in this respect were not well established.

Further author information: (Send correspondence to A.T.)

A.T., S.T. : E-mail: atokovinin, sthomas@ctio.noao.edu

G.V.: E-mail: gleb@okotech.com 
We hope that this paper clarifies the subject and will help to evaluate the MMDM technology for AO applications at large telescopes.

In Section 2 we present the properties of DM-79 and show that its real influence functions can be simulated using a thin-membrane equation. Section 3 addresses the problem of saturation and defines realistic saturation limits for curvature DMs. A modified non-linear control algorithm is introduced to alleviate the saturation effects. To conclude (Section 4), we give recommendations on the use of electrostatic DMs in astronomical AO systems.

\section{STUDY OF 50-MM DEFORMABLE MIRROR}

The 50-mm electrostatic DM-79 (Fig. 1) was developed at Okotech for the SOAR AO system. The 7-ring geometry of electrodes is given in Table 1, where the outer radius corresponds to the membrane edge and the angle $\phi$ is a rotation of each electrode ring with respect to some arbitrary reference. These angular offsets were selected to be random, in hope to improve the closed-loop control stability.

Table 1. Geometry of DM-79. The inner and outer radii of each ring $r_{\min }$ and $r_{\max }$ are given relative to the pupil radius, $N$ is the number of segments in each ring, and $\phi$ is the angular offset.

\begin{tabular}{|lcccc|}
\hline Ring & $r_{\min }$ & $r_{\max }$ & $N$ & $\phi$, degrees \\
\hline 1 & 0.00 & 0.165 & 1 & 0.0 \\
2 & 0.183 & 0.349 & 5 & 26.9 \\
3 & 0.367 & 0.532 & 11 & 27.7 \\
4 & 0.550 & 0.716 & 15 & 7.4 \\
5 & 0.734 & 0.899 & 17 & 8.4 \\
6 & 0.917 & 1.083 & 21 & 8.7 \\
7 & 1.101 & 1.529 & 9 & 3.0 \\
\hline
\end{tabular}
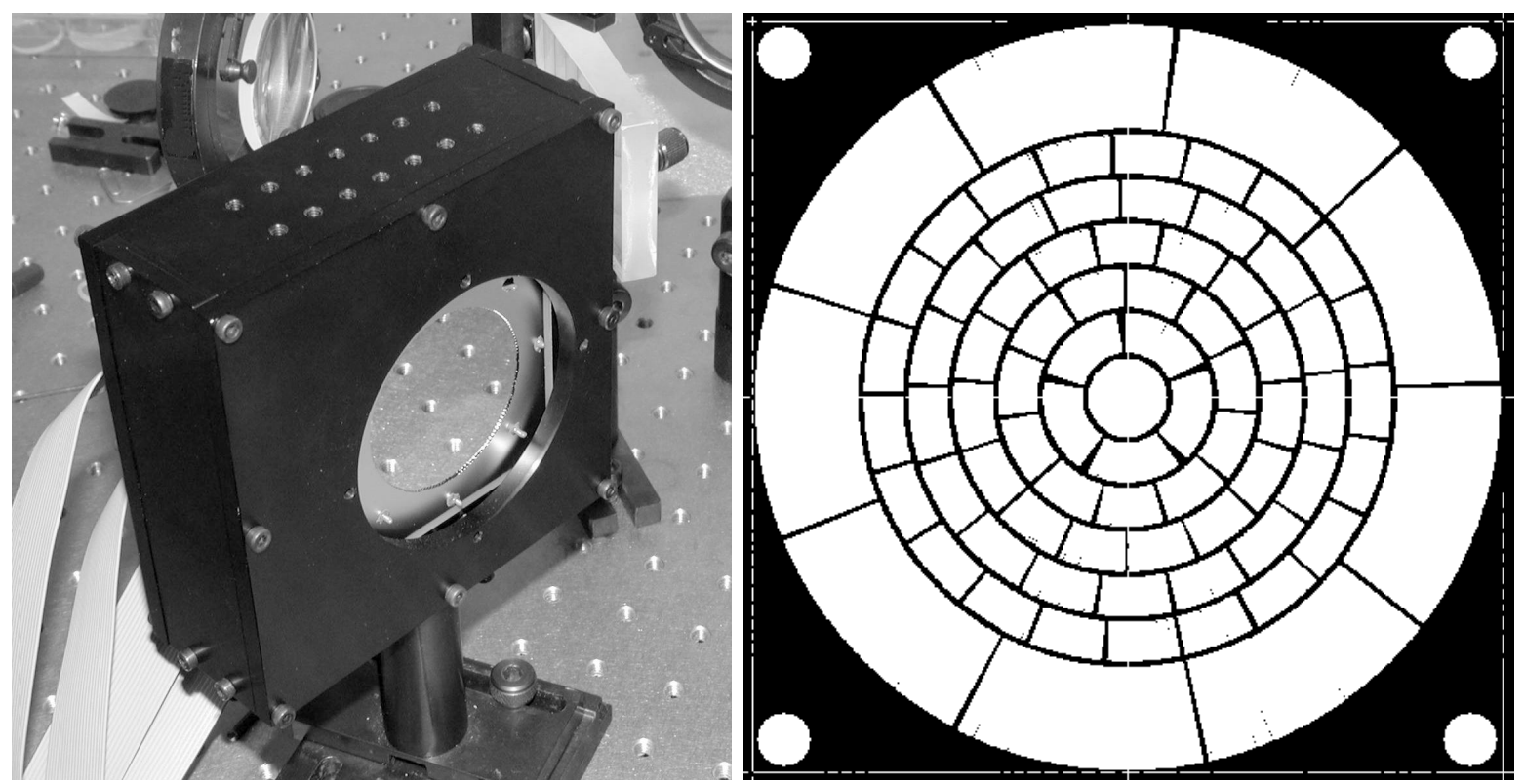

Figure 1. 79-channel 50-mm MMDM (left) and its electrode structure (right).

The high-voltage amplifiers provided with the DM give to the electrodes unipolar voltages in the range from 0 to $330 \mathrm{~V}$. When $V_{\max }=330 \mathrm{~V}$ is applied to all electrodes, the center of the membrane deflects by $28 \mu \mathrm{m}$. At 
small voltages $V$ the deflection is proportional to $V^{2}$, but as membrane deflects and gets closer to the electrodes the force increases faster than $V^{2}$.

In normal $\mathrm{AO}$ operation the $\mathrm{DM}$ is biased by applying $V_{\text {bias }}=V_{\max } / \sqrt{2}=233 \mathrm{~V}$ to all electrodes. The nominal shape of the DM is already curved, therefore any deformation of the DM should be considered relative to this biased state. Neglecting the deviation from the quadratic law, the "force" $f$ acting over each actuator is $f=\left(V^{2}-V_{\text {bias }}^{2}\right) / V_{\text {bias }}^{2}$ when a voltage $V$ is applied. The parameter $f$ changes from -1 (zero voltage, flat membrane) to +1 (maximum curvature), mapping linearly non-saturated range of control signals into $[-1,1]$ interval.

Because the border of the membrane is fixed, the deformation of the membrane is reasonably well modeled by a solution of the Poisson equation with fixed boundary condition. In discrete version this equation is

$$
4 z_{i j}-\left(z_{i-1, j}+z_{i+1, j}+z_{i, j-1}+z_{i, j+1}\right)=S f_{i j},
$$

where $z_{i j}$ is the deformation of the membrane at grid point $i, j$ and $f_{i j}$ is the normalized force acting at this point. The response coefficient $S$ scales the dimensionless factor $f$ to get the deformations $z$ in physical units.

The coefficient $S$ can be calculated by measuring the curvature radius of the DM in a biased state, $R_{\text {bias }}$. By setting $f$ to -1 or +1 , we change the DM curvature radius from infinity to $R_{b i a s} / 2$. The left-hand side of Eq. 1 - the Laplacian - correspondingly changes from zero to $4 p^{2} / R_{\text {bias }}$, where $p$ is the physical size of the grid pixels. At the same time the right-hand side changes by $2 S$, hence $S=2 p^{2} / R_{\text {bias }}$. If the curvature radius of the DM is replaced by the curvature radius of the wave-front (2 times less), the solutions of Eq. 1 will describe the wavefront deformation, 2 times larger.

We set $f_{i j}=1$ over some actuator and zero elsewhere and solve Eq. 1 by an iterative technique of simulated over-relaxation. ${ }^{7}$ It consists in adding the scaled difference of left- and right-hand parts of Eq. 1 to the previous iteration and zeroing the response at the membrane border. When the iterations converge, this difference tends to zero. A subtle point of the algorithm is that the order in which the loops over $i$ and $j$ are programmed influences the convergence.
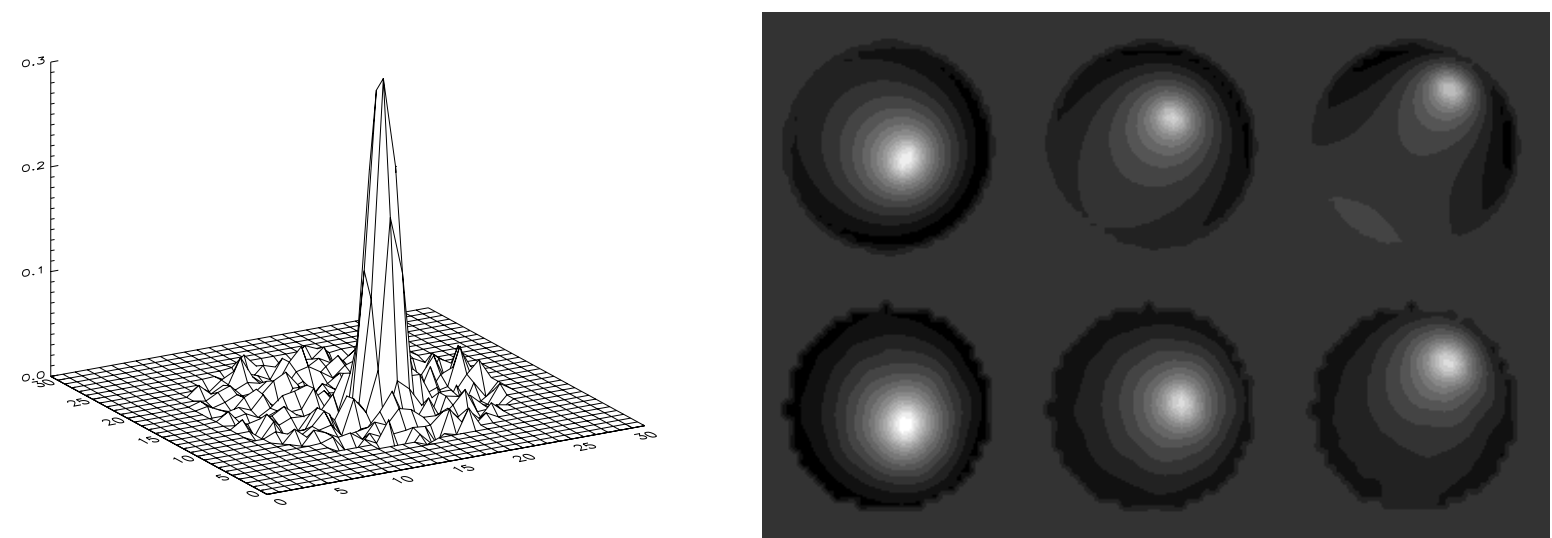

Figure 2. Left: Laplacian of the measured influence function for the electrode number 5. Right: The calculated (top row) and measured (bottom row) response of DM-79 for electrodes number 5, 16, and 48. All functions are displayed on the same intensity scale, the pupil diameter on DM is $38 \mathrm{~mm}$. Tip, tilt, and piston are subtracted.

We placed DM-79 in an optical setup and measured the deformation of its surface with the Wavescope wavefront sensor. ${ }^{8}$ The measured zone $38 \mathrm{~mm}$ in diameter was sampled by $25 \times 25$ sub-apertures. We applied a bias voltage $V_{\text {bias }}$ to all electrodes and considered the DM shape as reference. Then each electrode was set to zero voltage and the difference of the wave-front with respect to reference (i.e. the influence function) was measured. 
The piston and tip-tilt components are automatically subtracted from these functions by the Wavescope software. Three representative influence functions of DM-79 are displayed in Fig. 2 (bottom row).

We measured the DM curvature radius in biased state as $R_{\text {bias }}=25 \mathrm{~m}$, computed the calibration coefficient $S$ and solved Eq. 1 for each electrode. The solutions were processed in the same way as the measurements - restrict diameter to $38 \mathrm{~mm}$, subtract tip, tilt and piston. It should be stressed that no adjustment of amplitude was made. Yet, as we see in Fig. 2, the agreement between measured and modeled influence functions is impressive. We conclude that the shape of the real DM is well represented by a solution of the Poisson equation. All we need to know for computing the influence functions of this DM is the electrode geometry and the curvature radius $R_{\text {bias. }}$.

Table 2. First 15 Zernike amplitudes of DM-79 static aberrations (nm rms on wave-front) at different bias voltages.

\begin{tabular}{|ll|ccc|}
\hline Num. & Mode & $233 \mathrm{~V}$ & $0 \mathrm{~V}$ & $305 \mathrm{~V}$ \\
\hline 4 & focus & -87 & -3033 & 2900 \\
5 & 0-astigmatism & 377 & 337 & 426 \\
6 & 45-astigmatism & -95 & -74 & -122 \\
7 & x coma & 50 & 120 & -64 \\
8 & y coma & -18 & -46 & -3 \\
9 & x clover & 39 & 56 & 27 \\
10 & y clover & 24 & 8 & 41 \\
11 & spherical & 106 & 109 & 70 \\
12 & sph. ast. & -30 & -18 & -39 \\
13 & sph. ast. & -8 & -18 & 3 \\
14 & ashtray & -33 & -14 & -43 \\
15 & ashtray & -27 & -23 & -28 \\
\hline
\end{tabular}

Static aberrations of DM-79 were also studied with Wavescope for a pupil size of $32.6 \mathrm{~mm}$ (pupil border in the middle of the electrode ring 6). A flat wave-front was taken as reference. The decomposition of the measured wave-front on 15 first Zernike modes is given in Table 2 for 3 different bias voltages spanning the dynamic range of DM-79. The static aberrations are dominated by the astigmatism. The latter can be removed by adjusting the screws that hold the membrane-support structure. We fitted the first 15 Zernike modes of static aberrations with a combination of model influence functions in an attempt to determine which fraction of the DM dynamic will be consumed for self-compensation. If the astigmatism is removed by screw adjustment, a perfect compensation still needs actuator signals $f$ in the $[-0.39,+0.39]$ range, i.e. consumes some $39 \%$ of the DM dynamical range. If the astigmatism is to be compensated, the required range is $[-0.59,+0.58]$ and the rms wave-front residual after compensation is $10 \mathrm{~nm}$.

\section{SATURATION OF MEMBRANE DM}

Analytic estimate of the saturation level for a curvature DM is given in the book of Roddier, ${ }^{9}$ their Eq. 9.4:

$$
\epsilon_{\max }=8.8 \cdot 10^{4} \lambda^{-1 / 5} D^{-1} \Phi^{12 / 5} N^{-7 / 5}\left(2 / R_{W F}\right)^{6 / 5},
$$

where $\epsilon_{\max }$ is the seeing (FWHM in arcseconds) at wavelength $\lambda, D$ is the telescope diameter, $\Phi$ is the pupil diameter on the DM, $N$ is the number of actuators across the pupil (radial compensation order), and, finally, $R_{W F}$ is the maximum curvature radius of the wave-front at DM, assumed to be $1 / 3$ of the r.m.s. atmospheric curvature variation (clipping at $3 \sigma$ ). We repeated the derivation of this formula and found that $R_{W F}$ indeed refers to the wave-front, not to the DM as stated in the book. Straightforward application to the case of SOAR $\mathrm{AO}$ gives the maximum seeing that can be compensated as $\epsilon_{\max }=0.47^{\prime \prime}$ for $N=10$ and $\epsilon_{\max }=0.77^{\prime \prime}$ for $N=7$. Thus, DM-79 is at the limit of being acceptable, even without correcting its static aberrations. 

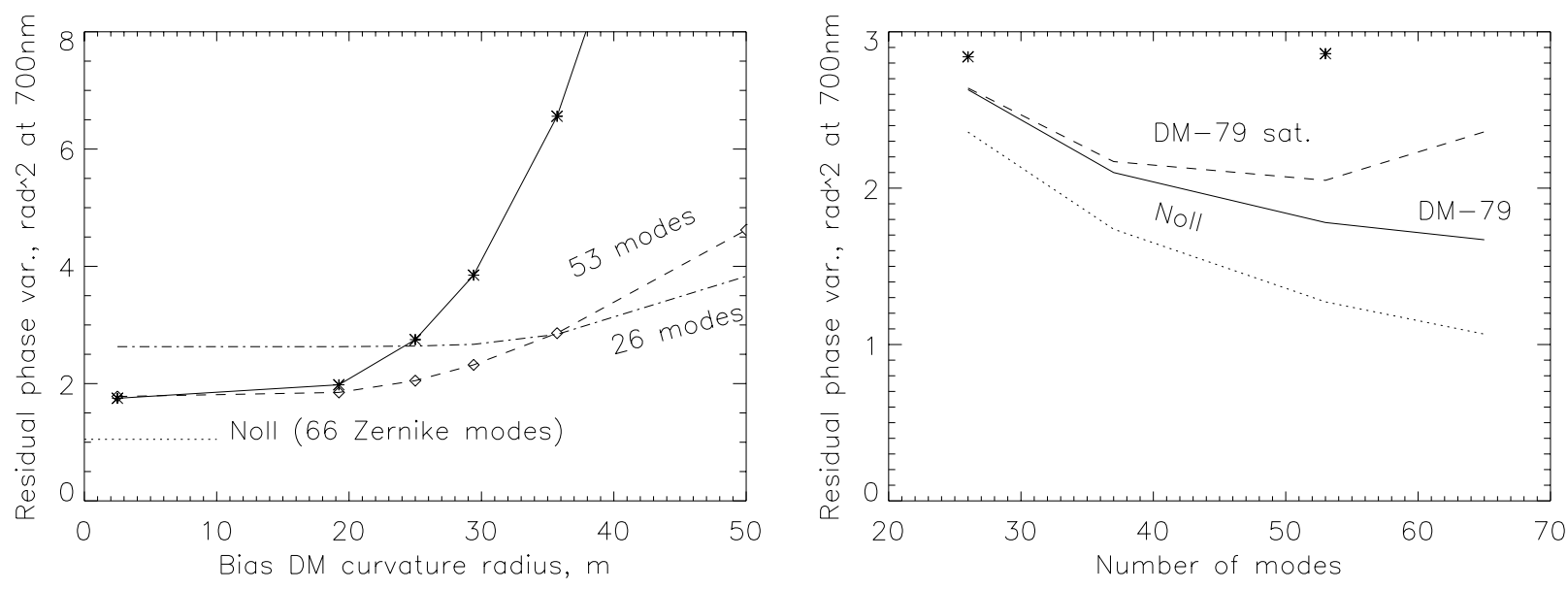

Figure 3. Left: Residual phase variance at median seeing as a function of the bias curvature radius of DM-79. Full line - linear control with 53 compensated system modes. Dashed and dash-dot lines - non-linear control with 53 and 26 compensated modes. Right: Residual phase variance at median seeing as a function of the number of corrected modes for Zernike polynomials (Noll), for DM-79 without saturation (full line), and for DM-79 with saturation and non-linear control (dashed). The two asterisks show the variance for dynamic range of the DM reduced by $30 \%$.

We used the results of our studies to simulate the behavior of DM-79 in our future AO system. The influence functions were calculated from the model. A 10x10 Shack-Hartmann wave-front sensor (SHWFS) was matched to DM-79 and an IDL Monte-Carlo simulation code (simul .pro by F. Rigaut) was run for $r_{0}=0.15 \mathrm{~m}$ at $500 \mathrm{~nm}$ wavelength and for telescope diameter $4.25 \mathrm{~m}$ (we now know that the actual SOAR mirror has useful diameter $4.1 \mathrm{~m}$ ). Tip and tilt are compensated by a separate mirror in these simulations.

In Fig. 3 (left) we plot in full line the residual phase variance as a function of the DM dynamic range, parametrized by the bias curvature radius. It is seen that the saturation of the real DM-79 $\left(R_{\text {bias }}=25 \mathrm{~m}\right)$ already increases the residual by $\sim 30 \%$ under median seeing. Any further decrease of dynamics has a catastrophic effect on the performance.

Roddier (Ref. 9, p. 212) states that curvature DMs are more tolerant to saturation than piezo-stack DMs: a saturation in curvature still permits to compensate the most important low-order (low-curvature) aberrations, hence the effect of saturation is less severe. Our results make it clear that saturation degrades the performance quite significantly. The apparent contradiction is possibly related to the fact that Roddier studied AO systems with a curvature wave-front sensor where there is a 1:1 correspondence between actuators and sensor elements. A saturated actuator distorts the signal in one sensor channel with little effect on adjacent channels. In our case a curvature DM is combined with a SHWFS, so each saturated actuator causes a large error signal in many SHWFS sub-apertures. This error signal propagates to other actuators at subsequent iterations of the AO loop, causing a substantial loss of performance.

Paterson et al. ${ }^{6}$ have already studied the saturation of a membrane DM and found that the best results in partially saturated regime are obtained by selecting a reduced number of controlled modes. By reducing the correction order $N$, we extend the saturation limit (Eq. 2) at the expense of leaving high-order modes uncorrected.

We found that a standard linear control of the AO system can be slightly improved in case of partial saturation. This modification, called non-linear control, is not computationally-intensive. Let the command vector $c$ obtained at some loop iteration be $c=c_{0}+t$, where $c_{0}$ is the clipped part (within the dynamic range) and $t$ is the truncated part. By construction, $t$ is different from zero only for saturated electrodes. We account for the missing signal, $t$, by applying some correction $d$ to non-saturated actuators: $c=c_{0}+d$. The most natural form is a linear combination of saturated signals, $d=D t$. The matrix $D$ re-distributes the "missing" part of the signal on saturated electrodes to the adjacent electrodes, it is related to the cross-talk matrix of the actuators. 
Fig. 3 shows that non-linear control significantly improves the results under partial saturation. The performance of DM-79 still degrades, but more slowly than for the linear control. Re-distribution of actuator signals is equivalent to reducing the correction order. Limiting the correction order by truncating weak system modes results in a worse residuals when there is no saturation but has little effect in the partially-saturated regime.

The dependence of residual phase variance on the number of corrected modes is shown explicitly in Fig. 3 (right). Even without saturation, the correction of $N$ system modes with DM-79 leaves larger residuals than the correction of the same number of Zernike modes - presumably because the combination of influence functions is not a perfect match to turbulence statistics. In presence of saturation, the phase residual is equivalent to correcting some 30 Zernike modes. If a dynamic range is reduced by $30 \%$ (e.g. for compensation of the static aberrations), the performance is further degraded.

Given that the saturation of DM-79 affects our AO system, we finally selected a bimorph mirror with $50 \mathrm{~mm}$ pupil size and modified the design of the AO instrument accordingly. Additional argument was the fragility of DM-79: the membrane cannot be touched, cleaned, etc., so the handling risk was estimated to be significant. Considering the purchase of the spare, the cost advantage of the electrostatic DM over a bimorph one became very modest.

\section{CONCLUSIONS}

The maximum curvature reached by electrostatic and bimorph DMs is comparable. Yet, bimorph DMs have a larger dynamic range and are used in $\mathrm{AO}$ systems at $8 \mathrm{~m}$ telescopes. The equation 2 explains why: the saturation threshold is a strong function of the pupil diameter at DM, $\Phi$. If membrane DMs could be made larger, they would be suitable for large telescopes. However, the size of the membrane is restricted by the fact that the bias deflection of the membrane is proportional to the square of the membrane diameter. For large membranes the

distance between the electrodes and the membrane should be made very large, which requires very high control voltages and makes the whole design very difficult to control.

Technologically, $50 \mathrm{~mm}$ mirror is the largest feasible MMDM and, according to our study, DM-79 can be used at telescopes with diameter of up to $3 \mathrm{~m}$, while DM-37 will be useful at telescopes up to $1.5 \mathrm{~m}$. Thus, MMDM is a viable and cheap option for $\mathrm{AO}$ systems at small and medium-sized telescopes.

\section{REFERENCES}

1. G. Vdovin, P.M. Sarro, "Flexible mirror micromachined in silicon", Appl. Opt., 34, pp. 2968-2972, 1995.

2. Ch. Keller, C. Plymate, S.M. Ammons, "Low-cost solar adaptive optics in the infrared", Proc. SPIE, 4853, pp. 351-359, 2003.

3. S. Restaino, G. Gilbreath, D. Payne, J. Baker, T. Martinez, M. DiVittorio, D. Mozurkewich, and J. Friedman, "Results from a portable Adaptive Optics system on the 1 meter telescope at the Naval Observatory Flagstaff Station", Proc, SPIE, 4838, pp. 1084-1090, 2003.

4. V.L. Krabbendam, T.A. Sebring, and S. Heathcote, "Southern Astrophysical Research Telescope (SOAR): steps on the road to success", Proc. SPIE, 4837, pp. 71-81, 2003.

5. S. Manhart, W.J. Hupfer, S. Nikolov, C. Wuerner, G.V. Vdovin, and Z. Sodnik, "50-mm MEMS deformable mirror", Proc. SPIE, 4007, pp. 555-562, 2000.

6. C. Paterson, I.H. Munro, and J.C. Dainty, "Low-cost adaptive optics system", Proc. SPIE, 4007, pp. 185-193, 2000.

7. W.H. Press, S.A. Teukolsky, W.T. Vetterling, and B.P. Flannery, Numerical Recipes in $C$, Cambridge Univ. Press, Cambridge, 1992, Sect. 17.

8. Adaptive Optics Associates, http://www.aoainc.com

9. F. Roddier, ed., Astronomical Adaptive Optics, Cambridge Univ. Press, Cambridge, 1999. 